\title{
DIMENSIONAMENTO E ESCALAS DE PESSOAL DE ENFERMAGEM: COMPETENNCIAS DOS ENFERMEIROS
}

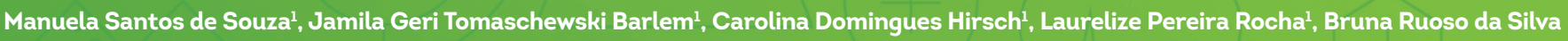
Neutzling ${ }^{1}$, Aline Marcelino Ramos ${ }^{1}$

Objetivo: analisar as competências necessárias para realização de dimensionamento e escalas de pessoal de enfermagem por enfermeiros. Metodologia: estudo quantitativo, do tipo exploratório-descritivo, realizado com 54 enfermeiros de um hospital universitário do sul do Brasil. A coleta de dados foi realizada através de um questionário estruturado autoaplicável, validado mediante validade de face, conteúdo e constructo. Para a análise de dados, utilizou-se a estatística descritiva e inferencial. Resultados: as atitudes foram identificadas como as competências mais importantes para a realização de dimensionamento e escalas de pessoal de enfermagem. Entre as habilidades, foi atribuida maior importância para a resolução de conflitos entre a equipe. Os enfermeiros se mostraram indiferentes quanto ao conhecimento dos custos gerados pelo cuidado. Conclusão: a análise das competências que fundamentam a realização do dimensionamento e de escalas de pessoal de enfermagem oportunizou o reconhecimento de importantes subsídios para qualificar a formação profissional e o trabalho da enfermagem.

DESCRITORES: Enfermagem, Recursos Humanos de Enfermagem, Administração de Recursos Humanos em Saúde.

\section{SIZING AND NURSES SCALES: SKILLS OF NURSES}

Objective: to analyze the skills required to perform nursing staff scales and scales. Methodology: a quantitative, exploratory-descriptive study with 54 nurses from a university hospital in the south of Brazil. Data collection was performed through a self-administered structured questionnaire, validated by face, content and construct validity. For data analysis, descriptive and inferential statistics were used. Results: the attitudes were identified as the most important skills for the accomplishment of scaling and scales of nursing personnel. Among the skills, greater importance was assigned to conflict resolution among the team. The nurses were indifferent about the knowledge of the costs generated by the care. Conclusion: the analysis of the competences that base the accomplishment of the dimensioning and nursing personnel scales allowed the recognition of important subsidies to qualify the professional training of nurses and nursing work.

Descriptors: Nursing, Nursing Staff, Health Personnel Management.

\section{DIMENSIONAMIENTO Y ESCALAS DE LAS ENFERMERAS: COMPETENCIAS DE LAS ENFERMEIRAS}

Objetivo: analizar las competencias necesarias para realizar el dimensionamiento y escalas para las enfermeras. Metodología: estudio cuantitativo de carácter exploratorio y descriptivo, realizado con 54 enfermeros de un hospital universitario en el sur de Brasil. La recolección de datos se llevó a cabo a través de un cuestionario administrado auto-estructurado, validado por la validez aparente, el contenido y la construcción. Para el análisis de datos se utilizó la estadística descriptiva e inferencial. Resultados: las actitudes han sido identificadas como las competencias más importantes para la realización de las escalas de diseño y enfermeras. Entre las habilidades, se le asigna mayor importancia a la resolución de conflictos entre el personal. Las enfermeras han demostrado ser indiferente al conocimiento de los costes derivados de atención. Conclusión: el análisis de las competencias que subyacen en la realización de las escalas de diseño y personal de enfermería proporcionados una oportunidad para reconocer las contribuciones importantes para calificar la formación de las enfermeras y el trabajo de enfermería.

Descriptores: Enfermería, Personal de Enfermería, Administración del Personal en Salud. 


\section{INTRODUÇÃO}

O processo de trabalho em saúde exige dos profissionais o desenvolvimento de determinadas competências, as quais compreendem conhecimentos, habilidades e atitudes que oportunizam a atuação desses profissionais nos diferentes espaços de assistência à saúde. Na enfermagem, tais competências são evidenciadas nas Diretrizes Curriculares Nacionais (DCN) para o Curso de Graduação em Enfermagem, compreendendo as seguintes competências e habilidades gerais: atenção à saúde, tomada de decisões, comunicação, liderança, administração e gerenciamento e educação permanente ${ }^{1}$.

Entende-se por competências os conhecimentos, habilidades e atitudes indispensáveis ao desenvolvimento das distintas atividades profissionais. O conhecimento é decorrente das experiências vivenciadas, da aprendizagem e da busca por informações. Por sua vez, as habilidades constituem aptidões desenvolvidas, determinando diferenças entre as pessoas em alguns aspectos. Já as atitudes decorrem de ações conscientes e de comportamentos associados à aplicação de princípios e valores²

Considerandotais conceitos, éimportanterefletire discutir a respeito das competências administrativas e gerenciais na formação dos enfermeiros, pois são questões que interferem diretamente na organização do trabalho da enfermagem(3). O enfermeiro tem importante atuação na construção e organização do quadro de pessoal de enfermagem, fazendose necessário verificar e analisar a formação qualitativa e quantitativa da equipe de enfermagem, tornando possivel a execução de uma assistência de enfermagem de maior qualidade ${ }^{4.5}$.

O dimensionamento de pessoal de enfermagem pode ser definido como um processo sistemático que subsidia o provimento de pessoal, tendo por finalidade prever e garantir a quantidade adequada de profissionais de enfermagem necessária para suprir as necessidades de cuidados de saúde(6). Atualmente, o dimensionamento de pessoal de enfermagem é sustentado legalmente pela Resolução COFEN 0527/2016, a qual evidencia que "compete ao Enfermeiro estabelecer o quadro quantiqualitativo de profissionais, necessário para a prestação da Assistência de Enfermagem" "7.

A partir da utilização de um Sistema de Classificação de Pacientes por complexidade assistencial (SCP), o dimensionamento de pessoal de enfermagem pode ser realizado conforme a dependência de assistência de enfermagem necessária, tendo como base o nível de complexidade dos cuidados ${ }^{8}$. Ainda no que se refere à administração e gerenciamento de pessoal de enfermagem, é competência do enfermeiro a realização das escalas de trabalho mensais e diárias de enfermagem ${ }^{9}$
O dimensionamento e a elaboração de escalas de pessoal de enfermagem são responsabilidades do enfermeiro e, para tanto, requerem determinadas competências gerenciais que irão subsidiar o provimento e a distribuição de pessoal necessário à prestação dos cuidados em saúde, o que justificou a realização desse estudo.

O presente estudo teve como questão de pesquisa: quais as competências necessárias para a realização de dimensionamento e escalas de pessoal de enfermagem por enfermeiros? E, como objetivo: analisar as competências necessárias para realização de dimensionamento e escalas de pessoal de enfermagem por enfermeiros.

\section{METODOLOGIA}

Estudo quantitativo, exploratório-descritivo, com delineamento transversal, realizado em um Hospital Universitário do Sul do Brasil, caracterizado como uma instituição pública, contando com a atuação de aproximadamente 70 enfermeiros. $O$ estudo foi realizado com uma amostra de 54 enfermeiros, selecionados mediante amostragem não probabilística por conveniência.

Para coleta de dados, realizada em outubro de 2015, foi elaborado um questionário estruturado autoaplicável construido com base na literatura sobre as competências necessárias para a realização de dimensionamento e elaboração de escalas de pessoal de enfermagem por enfermeiros(6,10-12) e validado mediante validade de face, conteúdo e constructo.

A escala, composta por 27 questões, objetiva medir o grau de importância dos conhecimentos, habilidades e atitudes necessários para realização do dimensionamento e elaboração de escalas de pessoal de enfermagem, sendo respondida por meio de uma escala de frequência do tipo Likert de cinco pontos. $\mathrm{O}$ instrumento apresenta, ainda, uma parte inicial de caracterização dos participantes.

Após os procedimentos relacionados aos aspectos éticos, os instrumentos foram entregues diretamente aos informantes em um envelope de papel pardo, sendo realizado o agendamento do recolhimento. O alfa de Cronbach do instrumento apresentou valor 0,92, enquanto que os coeficientes dos três constructos obtiveram os valores 0,80, 0,81 e 0,86, os quais são considerados satisfatórios para estudos exploratórios, confirmando a fidedignidade do instrumento.

A análise dos dados foi realizada a partir do software estatístico SPSS (Statistical Package for Social Sciences) versão 22.0, por meio de estatística descritiva e estatística inferencial. $O$ teste de Kolmogorov-Smirnov evidenciou que os dados possuem distribuição não paramétrica. Desse modo, foi utilizado o teste de Mann-Witney para comparação 
das médias de variáveis com duas categorias nominais.

Os aspectos éticos foram respeitados conforme as recomendações da Resolução 466/12 do Conselho Nacional de Saúde e o estudo foi aprovado pelo Comitê de Ética em Pesquisa local (Parecer n.136/2015).

\section{RESULTADOS}

Verificou-se que os 54 enfermeiros possuiam em média 39 anos $(39,69)$ e a maioria pertencia ao sexo feminino $(96,3 \%)$. A média de tempo de formação profissional foi de 13 anos $(13,9)$, variando de três meses até 35 anos de formados e, o tempo de atuação profissional variou entre um mês e 35 anos, sendo 13 anos $(13,62)$ a média de tempo de atuação profissional.

Quanto à realização de dimensionamento e elaboração de escalas de pessoal de enfermagem, 63,5\% afirmaram realizar dimensionamento de pessoal e $81,5 \%$ afirmaram que elaboram escalas. Quando questionados sobre o manuseio de sistemas informatizados para elaboração de escalas de pessoal de enfermagem, 74,1\% responderam que manuseiam tais sistemas e, desses, apenas 29,3\% afirmaram ter dificuldades.

Constatou-se que $92,6 \%$ dos participantes afirmaram ter obtido conhecimentos específicos direcionados à administração e gerenciamento de pessoal nos serviços de saúde durante a graduação em enfermagem. Quanto à realização da classificação de pacientes conforme o grau de dependência, 54,9\% referiu realizá-la. Contudo, 76,9\% respondeu não ter conhecimento acerca de qual escala de classificação de pacientes é utilizada na sua instituição de trabalho.

A análise descritiva (Tabela 1 ) permitiu verificar que o constructo atitudes necessárias apresentou a maior média do instrumento $(3,56)$, evidenciando que os enfermeiros consideram as atitudes como competências mais importantes pra realização de dimensionamento e escalas de pessoal de enfermagem, especialmente, quando se refere a ter liberdade na tomada de decisão e exercer a liderança $(3,76)$.

No constructo habilidades necessárias, a média correspondente foi igual a 3,47, assinalando que os enfermeiros consideram importante ter habilidades para realizar dimensionamento e escalas de pessoal de enfermagem. Nesse constructo, a questão "ser capaz de resolver conflitos entre a equipe na elaboração de escalas de pessoal de enfermagem" apresentou a maior média $(3,69)$.

O constructo conhecimentos necessários apresentou média 3,31, evidenciando que, de forma geral, os enfermeiros consideram que os conhecimentos são importantes para a realização de dimensionamento e escalas de pessoal de enfermagem. Contudo, a questão "ter conhecimento a respeito dos custos gerados pelo cuidado para realizar o dimensionamento de pessoal de enfermagem", apresentou média 2,80, evidenciando que os enfermeiros consideram que o conhecimento acerca de tais custos é indiferente para a realização do dimensionamento e elaboração de escalas de pessoal de enfermagem, considerando-os nem importantes, nem sem importância.

Tabela 1 - Média dos conhecimentos, habilidades e atitudes dos enfermeiros na realização de dimensionamento e escalas de pessoal de enfermagem - Rio Grande - 2015.

\begin{tabular}{|lcc|}
\hline \multicolumn{1}{|c}{ Fatores } & n & Média \\
\hline Conhecimentos & 54 & $(3,31)$ \\
\hline $\begin{array}{l}\text { q01 Ter conhecimento a respeito de administração de serviços } \\
\text { de saúde para realizar o dimensionamento e a elaboração de } \\
\text { escalas de pessoal de enfermagem. }\end{array}$ & 53 & 3,62 \\
$\begin{array}{l}\text { q02 Ter conhecimento a respeito da legislação trabalhista } \\
\text { para realizar o dimensionamento e a elaboração de escalas de } \\
\text { pessoal de enfermagem. }\end{array}$ & 54 & 3,48 \\
\hline
\end{tabular}

q03 Ter conhecimento a respeito dos sistemas informatizados para realizar o dimensionamento e a elaboração de escalas de $\quad 54 \quad 3,15$ pessoal de enfermagem.

q04 Ter conhecimento a respeito dos Sistemas de

Classificação de Pacientes para realizar o dimensionamento de $\quad 53 \quad 3,40$ pessoal de enfermagem.

q05 Ter conhecimento a respeito do tempo de assistência $\begin{array}{lll}\text { prestada e o esforço ocupacional, para realizar o } & 54 & 3,52\end{array}$ dimensionamento e a escala de pessoal de enfermagem

q06 Ter conhecimento a respeito dos custos gerados pelo

cuidado para realizar o dimensionamento de pessoal de $\quad 54 \quad 2,80$ enfermagem.

q07 Ter conhecimento a respeito das necessidades da instituição de saúde para realizar o dimensionamento e a $\quad 54 \quad 3,19$ escala de pessoal de enfermagem.

q08 Ter conhecimento a respeito das necessidades dos profissionais da equipe para realizar o dimensionamento e a escala de pessoal de enfermagem.

$54 \quad 3,41$

q09 Ter conhecimento a respeito das necessidades e particularidades dos pacientes para realizar o
dimensionamento e a escala de pessoal de enfermagem. Habilidades

q10 Ser capaz de raciocinar clinicamente acerca das condições dos pacientes para realizar o dimensionamento e a escala de pessoal de enfermagem.

qll Ser capaz de realizar a classificação de pacientes mediante aplicação de escalas específicas para realizar o dimensionamento de pessoal de enfermagem.

q12 Ser capaz de planejar, alocar, distribuir e supervisionar a equipe para realizar o dimensionamento e a escala de pessoal de enfermagem.

q13 Ser capaz de garantir a comunicação e a troca de informações claramente para realizar o dimensionamento e a escala de pessoal de enfermagem.

ql4 Ser capaz de garantir a comunicação e a troca de informações claramente para realizar o dimensionamento e a $\quad 54 \quad 3,57$ escala de pessoal de enfermagem.

q15 Ser capaz de comandar equipes na realização de escala de pessoal de enfermagem.

q16 Ser capaz de resolver conflitos entre a equipe na elaboração de escalas de pessoal de enfermagem.

q17 Ser capaz de realizar o dimensionamento e elaborar escalas de pessoal de enfermagem em sistemas informatizados.

Atitudes 


\begin{tabular}{|c|c|c|}
\hline $\begin{array}{l}\text { q18 Ser aberto ao diálogo na elaboração de escalas de pessoal } \\
\text { de enfermagem. }\end{array}$ & 54 & 3,65 \\
\hline $\begin{array}{l}\text { q19 Ser justo para realizar o dimensionamento e escalas de } \\
\text { pessoal de enfermagem. }\end{array}$ & 54 & 3,74 \\
\hline $\begin{array}{l}\text { q20 Ter autonomia para realizar o dimensionamento e escalas } \\
\text { de pessoal de enfermagem. }\end{array}$ & 54 & 3,74 \\
\hline $\begin{array}{l}\text { q21 Ter confiança para realizar o dimensionamento e escalas de } \\
\text { pessoal de enfermagem. }\end{array}$ & 54 & 3,57 \\
\hline $\begin{array}{l}\text { q22 Manter um bom relacionamento interpessoal para realizar } \\
\text { escalas de pessoal de enfermagem. }\end{array}$ & 54 & 3,52 \\
\hline $\begin{array}{l}\text { q23 Ter liberdade na tomada de decisão e exercer a liderança } \\
\text { para realizar o dimensionamento e escalas de pessoal de } \\
\text { enfermagem. }\end{array}$ & 54 & 3,76 \\
\hline $\begin{array}{l}\text { q24 Ser flexivel e considerar opinião dos membros da equipe } \\
\text { para realizar escalas de pessoal de enfermagem. }\end{array}$ & 54 & 3,43 \\
\hline $\begin{array}{l}\text { q25 Valorizar e entrosar-se com os membros da equipe } \\
\text { para realizar o dimensionamento e escalas de pessoal de } \\
\text { enfermagem. }\end{array}$ & 54 & 3,37 \\
\hline $\begin{array}{l}\text { q26 Estar aberto à inclusão de novas tecnologias para realizar } \\
\text { o dimensionamento e escalas de pessoal de enfermagem. }\end{array}$ & 54 & 3,39 \\
\hline $\begin{array}{l}\text { q27 Realizar reuniões e discussões com a equipe para realizar o } \\
\text { dimensionamento e escalas de pessoal de enfermagem. }\end{array}$ & 54 & 3,39 \\
\hline
\end{tabular}

Verificou-se que enfermeiros que não realizam o dimensionamento de pessoal atribuíram maior importância para as habilidades do que aqueles que o realizam. Também, foi possivel identificar que que enfermeiros que conheciam a escala de classificação de pacientes utilizada em sua instituição atribuíram maior importância aos conhecimentos, habilidades e atitudes para a realização de dimensionamento e escalas de pessoal de enfermagem (Tabela 2).

Tabela 2 - Relações entre as características da amostra e os constructos identificados através do teste de Mann-Whitney - Rio Grande, RS, 2015.

\begin{tabular}{|c|c|c|c|c|c|c|c|}
\hline & & \multicolumn{2}{|c|}{ Conhecimentos } & \multicolumn{2}{|c|}{ Habilidades } & \multicolumn{2}{|c|}{ Atitudes } \\
\hline & & M & $\mathrm{P}$ & M & $\mathrm{P}$ & M & $\mathrm{P}$ \\
\hline $\begin{array}{l}\text { Realiza } \\
\text { dimensionamento }\end{array}$ & $\mathrm{n}$ & & ,178 & 32,45 & $.030 *$ & 29,92 & 211 \\
\hline Não & 19 & 30,21 & & 23,08 & & 24,53 & \\
\hline Sim & 33 & 24,36 & & & & & \\
\hline $\begin{array}{l}\text { Conhece escala de } \\
\text { classificação }\end{array}$ & $n$ & & $.008^{*}$ & & $.002^{\star}$ & & $.012^{*}$ \\
\hline Não & 40 & 23,46 & & 23,00 & & 23,64 & \\
\hline Sim & 12 & 36,63 & & 38,17 & & 36,04 & \\
\hline
\end{tabular}

${ }^{\star} \mathrm{p}<0,05$ para significância estatística

\section{DISCUSSÃO}

Em relação à elaboração de escalas de pessoal de 54 | Enferm. Foco 2018; 9 (2): 50-55 enfermagem, a maior parte dos enfermeiros participantes desse estudo afirmou utilizar sistemas informatizados, não demonstrando dificuldades para o seu manuseio. A ampliação e aplicação de tais sistemas são indispensáveis para que o enfermeiro despenda de um tempo maior para dedicar-se a desenvolver suas atividades assistenciais ${ }^{6}$.

Quanto à realização da classificação de pacientes conforme o grau de dependência verificou-se que a maioria dos enfermeiros a realizam, entretanto, afirmaram não ter conhecimento acerca de qual escala de classificação de pacientes é utilizada na sua instituição de trabalho. Tal resultado vai ao encontro dos achados da literatura, uma vez que já foi verificado que no cotidiano de diferentes instituições de saúde o dimensionamento de pessoal tem sido realizado de forma empírica e fundamentado na experiência dos enfermeiros ${ }^{6}$.

No que se refere à avaliação das competências dos enfermeiros na realização de dimensionamento e escalas de pessoal de enfermagem, foi possivel perceber que os enfermeiros consideram as atitudes como competências mais importantes pra realização de dimensionamento e escalas de pessoal de enfermagem, especialmente, quando se refere a ter liberdade na tomada de decisão e exercer a liderança. Assim, em se tratando de algumas competências administrativas e gerenciais, os enfermeiros devem ter autoridade para a tomada de decisão, iniciativa e dinamismo, saber gerenciar e administrar tanto o ambiente de trabalho e sua organização, quanto os recursos estruturais, instrumentais e tecnológicos, bem como adquirir capacidade de empreendedorismo, gestão, chefia, lideranças e influência sobre a equipe de enfermagem ${ }^{1}$.

No que diz respeito às habilidades necessárias para realizar dimensionamento e escalas de pessoal de enfermagem, os enfermeiros desse estudo evidenciaram que as mesmas são importantes, especialmente, a capacidade de resolver conflitos entre a equipe. Na elaboração de escalas, é indispensável saber administrar as relações, pois nota-se que os conflitos estão predominantemente presentes no ambiente de trabalho e perante a diversidade dos indivíduos. Assim, percebe-se a importância do enfermeiro ter uma visão mais ampla em relação a sua equipe, conhecendo cada funcionário e suas ideias e desejos, tornando possivel um acordo na forma de divisão do trabalho junto com toda equipe ${ }^{10}$.

Os enfermeiros evidenciaram que os conhecimentos, de forma geral, são importantes para a realização de dimensionamento e escalas de pessoal de enfermagem, contudo desconhecem o reflexo dos custos desnecessários gerados pelo dimensionamento incorreto nesse processo. Com base nos achados da literatura, foi possivel observar que há um destaque para os custos com materiais utilizados na assistência prestada diretamente ${ }^{13,14}$. Entretanto, apesar de 
ser evidente a importância da realização de estudos acerca dos custos gerados na enfermagem, pouco se encontrou na literatura a respeito dos custos relacionados ao quadro quantitativo de pessoal, o que impossibilitou aprofundar as discussões dos resultados obtidos neste estudo.

Entre as relações encontradas acerca das características da amostra e as competências necessárias para realização de dimensionamento e escalas de pessoal de enfermagem, verificou-se que enfermeiros que não realizam o dimensionamento de pessoal atribuíram maior importância para as habilidades do que aqueles que o realizam. Nesse sentido, pode-se afirmar que os enfermeiros que não participam desse processo atribuem maior importância às ações que serão realizadas a partir do conhecimento obtido, uma vez que projetam suas expectativas nas habilidades que ainda não desenvolveram ${ }^{5}$.

Por fim, os enfermeiros participantes desse estudo que conheciam a escala de classificação de pacientes utilizada em sua instituição atribuíram maior importância aos conhecimentos, habilidades e atitudes para a realização de dimensionamento e escalas de pessoal de enfermagem. De acordo com alguns estudos, percebe-se a importância das escalas de classificação de pacientes, pois facilitam a mensuração do grau de dependência dos pacientes internados, auxiliando tanto na assistência de enfermagem, como na determinação da carga de trabalho nas unidades de internação ${ }^{15,16}$

Como limitações dessa pesquisa, destaca-se que ela foi conduzida em uma amostra específica de enfermeiros de uma instituição hospitalar do sul do Brasil, o que não permite a generalização dos seus resultados.

\section{CONCLUSÃO}

A análise dos conhecimentos, habilidades e atitudes que fundamentam a realização do dimensionamento e a elaboração de escalas de pessoal de enfermagem realizada por esse estudo oportunizou o reconhecimento de importantes subsidios para qualificar a formação profissional do enfermeiro e o processo de trabalho da enfermagem.

\section{REFERÊNCIAS}

1. Brasil. Conselho Nacional de Educação. Câmara de Educação Superior. Resolução CNE/CES 3/2001. Institui Diretrizes Curriculares Nacionais do Curso de Graduação em Enfermagem. Diário Oficial da União, Brasilia, 7 de Nov de 2001. Seção 1, p. 37.

2. Ruthes RM, Cunha ICKO. Competências do enfermeiro na gestão do conhecimento e capital intelectual. Rev Bras Enferm. 2009; 62(6):901-5.

3. Lourenção DCA, Benito GAV. Competências gerenciais na formação do enfermeiro. Rev Bras Enferm. 2010; 63(1):91-7.

4. Maya CM, Simões ALA. Implicações do dimensionamento do pessoal de enfermagem no desempenho das competências do profissional enfermeiro. Rev Bras Enferm. 2011; 64(5):508-14.

5. Oliveira RM, Leitao IMTA, Aguiar LL, Oliveira ACS, Gazos DM, Silva LMS, et al. Avaliando os fatores intervenientes na segurança do paciente: enfoque na equipe de enfermagem hospitalar. Rev Esc Enferm USP. 2015; 49(1):104-13.

6. Fugulin FMT, Gaidzinski RR, Castilho V. Dimensionamento de pessoal de enfermagem em instituições de saúde. In: Paulina Kurcgant. Gerenciamento em enfermagem. São Paulo: Guanabara Koogan; 2014

7. Conselho Federal de Enfermagem (COFEN). Resolução no 0527, de 03 de novembro de 2016. Atualiza e estabelece parâmetros para o dimensionamento do quadro de profissionais de Enfermagem nos serviços/locais em que são realizadas atividades de enfermagem. Rio de Janeiro: COFEN; 2016.

8. Urbanetto JS, Marco R, Carvalho SM, Creutzberg M, Oliveira KF, Magnago TBS. Grau de dependência de idosos hospitalizados conforme o sistema de classificação de pacientes. Rev Bras Enferm. 2012; 65(6):950-4
9. Juliani CMCM, Kurcgant P. Tecnologia educacional: avaliação de um website sobre escala de pessoal de enfermagem. Rev Esc Enferm USP. 2009; 43(3):512-9.

10. Ferreira FGKY, Martins CRM. Percepção de auxiliares e técnicos de enfermagem sobre a liderança organizacional de enfermeiros de um hospital do interior do Paraná. Publicatio UEPG. 2011; 19(2): 191-205.

11. Costa RA, Shimizu HE. Atividades desenvolvidas pelos enfermeiros nas unidades de internação de um hospital-escola. Rev Latino-am Enferm. 2005; 13(5):654-62.

12. Salvador PTCO, Oliveira RKM, Costa TD, Santos VEP, Tourinho FSV. Tecnologia e inovação para o cuidado em enfermagem. Rev Enferm UERJ. 2012; 20(1):111-7.

13. Inoue KC, Matsuda LM. Dimensionamento da equipe de enfermagem da UTI-adulto de um hospital ensino. Rev Eletrônica Enferm. 2009; 11(1):55-63.

14. Castilho V, Lima AFC, Fugulin FMT, Peres HHC, Gaidzinski RR. Custo total com pessoal para implementação de um sistema de apoio à decisão em enfermagem. Rev Latino-Am Enferm. 2014; 22(1):158-64.

15. Magalhães AMM, Riboldi CO, Guzinski C, Silva RC, Moura GMSS. Grau de dependência de pacientes em unidade de internação cirúrgica. Rev Bras Enferm. 2015; 68(5):542-7

16. Magalhães AMM, Dall'Agnol CM, Marck PB. Carga de trabatho da equipe de enfermagem e segurança do paciente - estudo com método misto na abordagem ecológica restaurativa. Rev Latino-Am Enferm. 2013; 21(esp):146-54. 